\title{
LOCAL ASYMPTOTICS OF \\ THE CYCLE MAXIMUM OF A \\ HEAVY-TAILED RANDOM WALK
}

\author{
DENIS DENISOV, ${ }^{*}$ EURANDOM \\ VSEVOLOD SHNEER, ${ }^{* *}$ Heriot-Watt University
}

\begin{abstract}
Let $\xi, \xi_{1}, \xi_{2}, \ldots$ be a sequence of independent and identically distributed random variables, and let $S_{n}=\xi_{1}+\cdots+\xi_{n}$ and $M_{n}=\max _{k \leq n} S_{k}$. Let $\tau=\min \left\{n \geq 1: S_{n} \leq 0\right\}$. We assume that $\xi$ has a heavy-tailed distribution and negative, finite mean $\mathrm{E}(\xi)<0$. We find the asymptotics of $\mathrm{P}\left\{M_{\tau} \in(x, x+T]\right\}$ as $x \rightarrow \infty$, for a fixed, positive constant $T$.

Keywords: Random walk; busy cycle; heavy-tailed distribution; stopping time; subexponential distribution

2000 Mathematics Subject Classification: Primary 60K25; 60G50

Secondary 60K30; 60G70
\end{abstract}

\section{Introduction and main result}

Let $\xi, \xi_{1}, \xi_{2}, \ldots$ be a sequence of independent random variables with a common distribution $F$ and mean $m,-\infty<-m<0$. Consider the random walk

$$
S_{0}=0, \quad S_{n}=\xi_{1}+\cdots+\xi_{n} .
$$

Let

$$
\tau=\min \left\{n \geq 1: S_{n} \leq 0\right\}, \quad M_{\tau}=\max _{0 \leq i \leq \tau} S_{i},
$$

be the first ladder epoch and the cycle maximum of the random walk, respectively. Note that, in this case, $\mathrm{E}(\tau)<\infty$ and $M_{\tau}<\infty$ almost surely. In this work we study the local asymptotics of the cycle maximum, i.e. the asymptotics of

$$
\mathrm{P}\left\{M_{\tau} \in(x, x+T]\right\}, \quad x \rightarrow \infty,
$$

where $T$ is a fixed, positive constant. We consider the heavy right tail case, i.e. in which $\mathrm{E}\left(\mathrm{e}^{\lambda \xi_{1}}\right)=\infty$ for all $\lambda>0$.

Random walks with heavy-tailed increments have been the subject of many studies. Besides their own intrinsic interest, they have many applications in queueing theory, risk theory, etc. The subject of interest in this paper is (the local tail asymptotics of) the busy cycle maximum. This quantity plays an important role in the analysis of the single-server queue. For example, the (global) tail asymptotics of the busy cycle maximum has been applied to find the tail asymptotics of the busy period of the GI/GI/1 queue; see [17]. In turn, the busy period has many

Received 23 September 2005; revision received 4 September 2006.

* Postal address: EURANDOM, PO Box 513, 5600 MB Eindhoven, The Netherlands.

Email address: denisov@eurandom.tue.nl

** Current address: EURANDOM, PO Box 513, 5600 MB Eindhoven, The Netherlands.

Email address: shneer@eurandom.tue.nl 
applications to various other problems, for example generalized processor sharing, priority queues, convergence rates in queueing problems, and the sojourn time in the GI/GI/1 queue with the last-came-first-served discipline. It may also be used to find the (global tail asymptotics of the) sojourn time in the GI/GI/1 processor-sharing queue. Therefore, we expect that the present paper will be useful in obtaining local versions of the above results.

The global asymptotics of $\mathrm{P}\left\{M_{\tau}>x\right\}$ (and some related problems) has been studied by various authors. In [13] this asymptotics was obtained for regularly varying distributions. In [1] (see also corrections in the proof of [2, Theorem X.9.4]) this asymptotics was found for a more general class, $f^{*}$ (see Definition 1, below): it was proved that if $F$ belongs to $\delta^{*}$ then

$$
\mathrm{P}\left\{M_{\tau}>x\right\} \sim \mathrm{E}(\tau) \bar{F}(x)
$$

(here and throughout, $a(x) \sim b(x)$ is written to mean that $\lim _{x \rightarrow \infty} a(x) / b(x)=1$, and for any distribution function $F$ we let $\bar{F}(x):=1-F(x))$. A short proof of (1) may be found in [8]. Foss and Zachary [11] showed that the converse is true: if $F$ is long tailed and (1) holds, then $F \in 8^{*}$. They also proved that (1) holds even if we assume that $\tau$ is an arbitrary stopping time with finite mean. In [12] this result was generalized to the case of infinite-mean stopping times.

In order to state our results we require some definitions.

Definition 1. A distribution function $F$ on $\mathbb{R}$ belongs to the class $8^{*}$ (see [14]) if and only if $\bar{F}(x)>0$ for all $x$ and

$$
\int_{0}^{x} \bar{F}(x-y) \bar{F}(y) \mathrm{d} y \sim 2 m^{+} \bar{F}(x),
$$

where $m^{+}=\int_{0}^{\infty} \bar{F}(y) \mathrm{d} y<\infty$.

It is known that if a distribution function $F$ belongs to the class $8^{*}$, then it is subexponential (see [14]). In general, the converse assertion does not hold, i.e. a subexponential distribution with finite mean may not belong to $8^{*}$; see [9] for an example of this.

Fix a $T, 0<T \leq \infty$, and write $\Delta=(0, T]$ and

$$
x+\Delta=(x, x+T], \quad x \in \mathbb{R} .
$$

Let

$$
F(x+\Delta)=\mathrm{P}\{\xi \in x+\Delta\}=\mathrm{P}\{\xi \in(x, x+T]\} .
$$

Definition 2. We say that a distribution $F$ on $\mathbb{R}$ belongs to the class $\mathcal{L}_{\Delta}$ if and only if $F(x+\Delta)>$ 0 for all sufficiently large $x$ and, for all $t \in[0,1]$,

$$
\frac{F(x+t+\Delta)}{F(x+\Delta)} \rightarrow 1 \quad \text { as } x \rightarrow \infty .
$$

Remark 1. The class $\mathcal{L}_{\Delta}$ was introduced in [3]. Note that Definition 2 implies local uniform convergence (uniform convergence on each compact set of $t$ in $(0, \infty)$ ) in (2). Indeed, it follows from Definition 2 that (2) holds for all $t \geq 0$. Let $f(x)=F(\log x+\Delta)$; then (2) is equivalent to $f(t x) / f(x) \rightarrow 1$ as $x \rightarrow \infty$. This means that the function $f$ is slowly varying (see [5] for the relevant definition and properties). Uniform convergence in (2) follows now from the uniform convergence theorem for slowly varying functions (see, e.g. [5, Theorem 1.2.1]). Moreover, it follows from the uniform convergence on any compact set that we can choose a function $h(x) \rightarrow \infty$ such that (2) holds uniformly in $|t| \leq h(x)$. 
Definition 3. Let $F$ be a distribution on $\mathbb{R}_{+}$with unbounded support. We say that $F$ is $\Delta$-subexponential, and write $F \in \varsigma_{\Delta}$, if $F \in \mathscr{L}_{\Delta}$ and

$$
(F * F)(x+\Delta) \sim 2 F(x+\Delta) \quad \text { as } x \rightarrow \infty .
$$

If $T=\infty$ then we simply say that $F$ is subexponential.

The notion of $\Delta$-subexponential distributions was introduced in [3]. The case in which $T=\infty$ corresponds to that of subexponential distributions introduced by Chistyakov [7]. In [3] it was shown that the basic properties of subexponential distributions carry over virtually without change to the case of $\Delta$-subexponential distributions.

In this paper we introduce a new class of distributions.

Definition 4. We say that a distribution $F$ belongs to the class $\delta \tau_{\Delta}^{*}$ if $F \in \mathcal{L}_{\Delta}, m^{+}<\infty$, and

$$
\int_{0}^{x / 2} F(x-y+\Delta) F((y, x]) \mathrm{d} y \sim m^{+} F(x+\Delta) \quad \text { as } x \rightarrow \infty .
$$

This class is an extension of the class $\delta^{*}$ introduced in [14]. It is not difficult to see that $\delta^{*}=\delta_{(0, \infty)}^{*}$ since $\varsigma^{*} \subset \mathcal{L}_{(0, \infty)}$ (see [14]). We will also show that if $F$ belongs to $\delta \tau_{\Delta}^{*}$ for some $\Delta$, then it belongs to $\delta_{\Delta}$ as well.

Now we are in position to state our main result.

Theorem 1. (i) Suppose that $F \in \delta \tau_{\Delta}^{*}$. Then

$$
\mathrm{P}\left\{M_{\tau} \in x+\Delta\right\} \sim \mathrm{E}(\tau) F(x+\Delta), \quad x \rightarrow \infty .
$$

(ii) Conversely, suppose that the asymptotics (3) holds and that $F \in \mathcal{L}_{\Delta}$. Then $F \in \delta \tau_{\Delta}^{*}$.

The proof of Theorem 1 is given in Section 3 .

Foss and Zachary [11] gave a natural extension of (1) to the case of a general stopping time $\sigma$ : they showed that, for an arbitrary stopping time $\sigma$ with finite mean, $\mathrm{P}\left\{M_{\sigma}>x\right\} \sim \mathrm{E}(\sigma) \bar{F}(x)$. In the local case such an extension of Theorem 1 does not hold in general. In Section 5 we show that for any $F \in \mathcal{L}_{\Delta}$ there exists a finite-mean stopping time $\sigma$ such that

$$
\limsup _{x \rightarrow \infty} \frac{\mathrm{P}\left\{M_{\sigma} \in x+\Delta\right\}}{F(x+\Delta)}>\mathrm{E}(\sigma) .
$$

The paper is organized as follows. In Section 2, in the form of nine lemmas, we present some properties of the new class, $\delta \tau_{\Delta}^{*}$. We show that the main properties of the class $\delta^{*}$ remain valid for the case of an arbitrary, positive $T$. We also give sufficient conditions for a distribution to belong to the class $\delta \tau_{\Delta}^{*}$. Using these sufficient conditions we show that standard examples of subexponential distributions are contained in the class $\delta \tau_{\Delta}^{*}$. The proof of Theorem 1 is given in Section 3. In Section 4 we prove Theorem 2, which gives the asymptotics of the taboo renewal function (see (11) for the definition). In Section 5 we construct a stopping time $\sigma$ for which strict inequality (4) holds. Proofs of the nine lemmas formulated in Section 2 are collected in the appendix. 


\section{Basic properties of the class $\& \tau_{\Delta}^{*}$}

We start with a lemma which gives equivalent definitions of the class $\delta \tau_{\Delta}^{*}$.

Lemma 1. Let $F \in \mathcal{L}_{\Delta}$ and let $h(x) \uparrow \infty$, with $h(x) \leq x / 2$ being a function such that $F(x-y+\Delta) \sim F(x+\Delta)$ uniformly in $y \leq h(x)$. Then

$$
\begin{aligned}
F \in \delta \tau_{\Delta}^{*} & \Longleftrightarrow \int_{h(x)}^{x / 2} F((y, x]) F(x-y+\Delta) \mathrm{d} y=o(F(x+\Delta)) \\
& \Longleftrightarrow \int_{h(x)}^{x-h(x)} F((y, x]) F(x-y+\Delta) \mathrm{d} y=o(F(x+\Delta)) \\
& \Longleftrightarrow \int_{0}^{x-h(x)} F((y, x]) F(x-y+\Delta) \mathrm{d} y \sim m_{+} F(x+\Delta) .
\end{aligned}
$$

In addition, $F \in \delta \tau_{\Delta}^{*}$ implies that

$$
\int_{0}^{x} F(x-y+\Delta) F((y, x]) \mathrm{d} y=O(1) F(x+\Delta) .
$$

We give some conditions for a distribution to belong to the class $\delta \tau_{\Delta}^{*}$. These conditions show that standard examples of subexponential distributions are contained in the class $\delta \tau_{\Delta}^{*}$.

Lemma 2. Let a distribution $F$ belong to the class $\mathcal{L}_{\Delta}$. Assume that there exist $c>0$ and $x_{0}<\infty$ such that $F(x+t+\Delta) \geq c F(x+\Delta)$ for any $x>x_{0}$ and $t \in(0, x]$. Assume also that $m^{+}<\infty$. Then $F \in \delta \tau_{\Delta}^{*}$.

Remark 2. In [3] it was shown that if a distribution $F$ satisfies the conditions of Lemma 2, then $F \in s_{\Delta}$. It is clear that $\bar{F}(2 x) \geq c \bar{F}(x)$ for such distributions, and it was shown in [14, Theorem 3.2] that distributions with this property belong to the class $\delta^{*}$.

The Pareto distribution (with the tail $\bar{F}(x)=x^{-\alpha}, \alpha>1, x \geq 1$ ) satisfies the conditions of Lemma 2 for any $T>0$. The same is true for any distribution $F$ such that $\mathrm{P}\{\xi \in x+\Delta\}$ is regularly varying at infinity, i.e. for any distribution $F(x+\Delta) \sim x^{-\alpha} l(x)$ where $l(x)$ is slowly varying at infinity.

Let $Q_{\Delta}(x)=-\ln F(x+\Delta)$ for any finite $T$, and let $Q(x)=-\ln \bar{F}(x)$. Following, with obvious changes, the construction presented in [14] (see also [16]), it is easy to check that for any distribution $F \in \mathcal{L}_{\Delta}$ we can always find a distribution $G$ such that $G \in \mathcal{L}_{\Delta}$, $F(x+\Delta) \sim G(x+\Delta)$ as $x \rightarrow \infty$, and $R_{\Delta}(x):=-\ln G(x+\Delta)$ is differentiable. In view of Lemma 9, below, we may give sufficient conditions for $F \in \mathcal{s}_{\Delta}^{*}$, assuming the existence of the derivative $Q_{\Delta}^{\prime}(x)$.

Lemma 3. Assume that $r:=\lim _{\sup _{x \rightarrow \infty}} x Q_{\Delta}^{\prime}(x) / Q(x)<1$, that the function $Q(x) / x$ is eventually nonincreasing, and that $\bar{F}^{1-r-\varepsilon}(x)$ is integrable for some $\varepsilon>0$. Then $F \in \delta \tau_{\Delta}^{*}$.

Remark 3. Lemma 3 is a generalization of [15, Theorem 2.8(c)] to the case of an arbitrary, positive $T$. Note that, in the case $T=\infty$, the conditions of Lemma 3 and [15, Theorem 2.8(c)] coincide, since in this case the fact that $Q(x) / x$ is a nonincreasing function follows from the assumption that $r<1$.

Direct computations show that any Weibull distribution (i.e. any distribution with the tail $\bar{F}(x)=\mathrm{e}^{-x^{\gamma}}$ ) satisfies the conditions of Lemma 3 for any $T>0$ if $0<\gamma<1$. It can also 
be shown that so-called semiexponential distributions (i.e. distributions with the tails $\bar{F}(x)=$ $\mathrm{e}^{-x^{\gamma} l(x)}$ where $0 \leq \gamma<1$ and $l(x)$ is a slowly varying function such that $l^{\prime}(x)=o(l(x) / x)$ as $x \rightarrow \infty$; see, e.g. [6]) satisfy the conditions of Lemma 3 for any $T>0$.

The next two lemmas will be extensively used in the proof of Theorem 1. Lemma 4 shows that $F((y, x])$ behaves like a long-tailed function.

Lemma 4. Let $F \in \mathcal{L}_{\Delta}$ with a finite $T \geq 0$. For any $t>0$ and $\varepsilon>0$, there exists a $y_{0}>0$ such that, for all $x$ and $y$ with $y \in\left(y_{0}, x-y_{0}\right)$,

$$
\frac{F((y-t, x+t])}{F((y, x])} \leq 1+\varepsilon .
$$

Lemma 5 shows that $F((y, x])$ behaves like a distribution function from $8^{*}$.

Lemma 5. Let $F \in 8 \tau_{\Delta}^{*}$ with a finite $T \geq 0$. Then, for any $\varepsilon>0$, there exists a $y_{0}>0$ such that, for all $x$ and $y$ with $y \in\left(y_{0}, x-y_{0}\right)$,

$$
\int_{y_{0}}^{y-y_{0}} F((y-u, x-u]) F((u, x]) \mathrm{d} u \leq \varepsilon F((y, x])
$$

and

$$
2 m_{+}-\varepsilon \leq \frac{\int_{0}^{y} F((y-u, x-u]) F((u, x]) \mathrm{d} u}{F((y, x])} \leq 2 m_{+}+\varepsilon .
$$

If $T=\infty$ then Lemma 4 and Lemma 5 hold for $x=\infty$. The property in the next lemma is an analogue of a property of $s_{\Delta}$.

Lemma 6. If $F \in \delta \tau_{\Delta}^{*}$ for some finite interval $\Delta=(0, T]$, then $F \in \delta \tau_{\Delta}^{*}$ for any $\Delta=$ $(0, n T], n \in \mathbb{N}$.

It is known (see [3]) that $\varsigma_{\Delta} \subset S$ for any positive $T$. Lemma 7 shows that the inclusion $8 \tau_{\Delta}^{*} \subset 8^{*}$ also holds.

Lemma 7. If $F \in \& \tau_{\Delta}^{*}$ for some finite interval $\Delta=(0, T]$, then $F \in \varsigma^{*}$.

It is also known (see [14]) that $\varsigma^{*} \subset S$. Lemma 8 shows that the inclusion $\diamond \tau_{\Delta}^{*} \subset \diamond_{\Delta}$ also holds.

Lemma 8. If $F \in \delta \tau_{\Delta}^{*}$ for some finite interval $\Delta=(0, T]$, then $F \in \varsigma_{\Delta}$.

The following lemma is a generalization of [14, Theorem 2.1(b)] to the case of an arbitrary positive $T$.

Lemma 9. Let $F, G \in \mathcal{L}_{\Delta}$ and assume that there exist $M_{1}, M_{2} \in(0, \infty)$ such that $M_{1} \leq$ $G(x+\Delta) / F(x+\Delta) \leq M_{2}$ for all sufficiently large $x$. Then $F \in s \tau_{\Delta}^{*}$ if and only if $G \in s \tau_{\Delta}^{*}$.

Remark 4. One might wonder whether the class $\delta \tau_{\Delta}^{*}$ is equal to another class generalizing $\delta^{*}$. We say that a distribution $F$ belongs to the class $\wp_{\Delta}^{*}$ if $F \in \mathcal{L}_{\Delta}, m^{+}<\infty$, and

$$
\int_{0}^{x / 2} F(x-y+\Delta) \bar{F}(y) \mathrm{d} y \sim m^{+} F(x+\Delta) \quad \text { as } x \rightarrow \infty .
$$

The definition of this class almost coincides with that of $\delta \tau_{\Delta}^{*}$. Lemma 1 immediately implies that $\delta_{\Delta}^{*} \subset \delta \tau_{\Delta}^{*}$. The authors do not know whether or not the converse inclusion holds, but we have some reasons to believe that $\delta_{\Delta}^{*} \neq \delta \tau_{\Delta}^{*}$. The results of this section all hold for $\delta_{\Delta}^{*}$ as well. 


\section{Proof of Theorem 1}

We first give several definitions. Let $M=\sup _{n \geq 0} S_{n}$ and $\pi(B)=\mathrm{P}\{M \in B\}$. An important role in this work is played by the taboo renewal function defined as follows:

$$
H_{x}(B) \equiv \sum_{n=0}^{\infty} \mathrm{P}\left\{\tau>n, M_{n} \leq x, S_{n} \in B\right\} .
$$

Clearly, $H_{x}(B)$ is monotone increasing in $x$ and $H_{\infty}(B)=\mathrm{E}(\tau) \pi(B)$; see, e.g. [2, Chapter VIII, Theorems 2.2 and 2.3] for the latter equality. Our main tool is the following theorem, which will be proved in Section 4.

Theorem 2. Let $F \in \delta \tau_{\Delta}^{*}$. Let $b>0$ be any number if $F$ is a nonlattice distribution, and let $b=n h, n \in \mathbb{N}$, if $F$ is a lattice distribution with step size $h$. For any $\varepsilon>0$, there exists a $y_{0}>0$ such that, for $x$ and $y$ with $y \in\left(y_{0}, x-y_{0}\right)$,

$$
(1-\varepsilon) \mathrm{E}(\tau) \frac{b}{m} F((y, x]) \leq H_{x}((y, y+b]) \leq(1+\varepsilon) \mathrm{E}(\tau) \frac{b}{m} F((y, x]) .
$$

For the lower bound to hold it is sufficient to assume that $F \in \mathcal{L}_{\Delta}$.

Let $\mu(x)=\min \left\{n \geq 1: S_{n}>x\right\}$ be the time the random walk first exceeds the level $x$. Our starting point is the following asymptotic representation.

Lemma 10. Let $A>0$ be any constant. Then, uniformly in $a \in[0, A]$,

$$
\begin{aligned}
\mathrm{P}\left\{M_{\tau}\right. & \in x+\Delta\} \\
& =(1+o(1)) \mathrm{P}\left\{S_{\mu(x-a)}+\tilde{M} \in x+\Delta, \mu(x-a) \leq \tau\right\} \\
& =(1+o(1)) \int_{0}^{x-a} H_{x-a}(\mathrm{~d} y) \mathrm{P}\{\tilde{M}+\xi \in x-y+\Delta, \xi \in(x-y-a, x-y+T]\}
\end{aligned}
$$

where $\tilde{M} \stackrel{\mathrm{D}}{=} M$ is independent of $\left\{\xi_{n}\right\}_{n \geq 1}$.

Proof. For any $a>0$,

$$
\begin{aligned}
\mathrm{P}\left\{M_{\tau}\right. & \in x+\Delta\} \\
& =\mathrm{P}\left\{\mu(x-a) \leq \tau, \max _{\{i: \mu(x-a) \leq i \leq \tau\}} S_{i} \in x+\Delta\right\} \\
& =\mathrm{P}\left\{\mu(x-a) \leq \tau, S_{\mu(x-a)} \in(x-a, x+T], \max _{\{i: \mu(x-a) \leq i \leq \tau\}} S_{i} \in x+\Delta\right\} \\
& =\int_{x-a}^{x+T} \mathrm{P}\left\{\mu(x-a) \leq \tau, S_{\mu(x-a)} \in \mathrm{d} z, \max _{\{i: \mu(x-a) \leq i \leq \tau\}}\left(S_{i}-S_{\mu(x-a)}\right) \in x-z+\Delta\right\} .
\end{aligned}
$$

Making use of the Markov property, we can represent the latter equality as

$$
\mathrm{P}\left\{M_{\tau} \in x+\Delta\right\}=\int_{x-a}^{x+T} \mathrm{P}\left\{\mu(x-a) \leq \tau, S_{\mu(x-a)} \in \mathrm{d} z\right\} \mathrm{P}\left\{\tilde{M}_{\tilde{\tau}_{-z}} \in x-z+\Delta\right\},
$$

where $\tilde{\tau}_{-z}=\inf \left\{n \geq 1: \tilde{S}_{n} \leq-z\right\}, \tilde{M}_{n}=\max _{0 \leq i \leq n} \tilde{S}_{i}$, and $\tilde{S}_{n}$ is a random walk independent of $\left\{\xi_{n}\right\}_{n=1}^{\infty}$. It is not difficult to see that, uniformly in $u \in[-T, A]$,

$$
\mathrm{P}\left\{\tilde{M}_{\tilde{\tau}_{-z}} \in u+\Delta\right\} \rightarrow \mathrm{P}\{\tilde{M} \in u+\Delta\}, \quad z \rightarrow \infty .
$$


Therefore, uniformly in $a \in[0, A]$,

$$
\begin{aligned}
\mathrm{P}\left\{M_{\tau} \in x+\Delta\right\} & =(1+o(1)) \int_{x-a}^{x+T} \mathrm{P}\left\{\mu(x-a) \leq \tau, S_{\mu(x-a)} \in \mathrm{d} z\right\} \mathrm{P}\{\tilde{M} \in x-z+\Delta\} \\
& =(1+o(1)) \mathrm{P}\left\{\tilde{M}+S_{\mu(x-a)} \in x+\Delta, \mu(x-a) \leq \tau\right\}, \quad x \rightarrow \infty .
\end{aligned}
$$

Our next step is to prove (13). By the total probability formula, for any measurable $B \subset$ $(x, \infty)$, we have

$$
\begin{aligned}
\mathrm{P}\left\{\mu(x) \leq \tau, S_{\mu(x)} \in B\right\} & =\sum_{n=1}^{\infty} \mathrm{P}\left\{\tau \geq n, M_{n-1} \leq x, S_{n} \in B, \mu(x)=n\right\} \\
& =\int_{0}^{x} \sum_{n=1}^{\infty} \mathrm{P}\left\{\tau \geq n, M_{n-1} \leq x, S_{n-1} \in \mathrm{d} y\right\} F(B-y) \\
& =\int_{0}^{x} H_{x}(\mathrm{~d} y) F(B-y) .
\end{aligned}
$$

After substituting this expression into (12), we obtain (13).

Since the asymptotic equivalence in (13) holds uniformly in any interval $[0, A]$, it is possible to pick a function $h(x) \uparrow \infty$ with $h(x)<x / 2$ such that (13) holds uniformly in $a \in[0, h(x)]$. In addition, since $F \in \mathcal{L}_{\Delta}$, we can pick this function $h(x)$ to satisfy

$$
\sup _{y \leq 2 h(x)}\left|\frac{F(x-y+\Delta)}{F(x+\Delta)}-1\right| \rightarrow 0 .
$$

Lemma 11. Let $F \in \mathcal{L}_{\Delta}$. For the function $h(x)$ defined above and any function $a(x) \uparrow \infty$,

$$
\begin{aligned}
\int_{0}^{h(x)} H_{a(x)}(\mathrm{d} y) \mathrm{P}\{\tilde{M}+\xi \in x-y+\Delta, \xi \in(x-y-h(x), x-y+T]\} & \\
& \sim \mathrm{E}(\tau) F(x+\Delta) .
\end{aligned}
$$

Lemma 12. Let $F \in s \tau_{\Delta}^{*}$. For the function $h(x)$ defined above,

$$
\begin{array}{r}
\int_{h(x)}^{x-h(x)} H_{x-h(x)}(\mathrm{d} y) \mathrm{P}\{\tilde{M}+\xi \in x-y+\Delta, \xi \in(x-y-h(x), \\
x-y+T]\} \\
=o(F(x+\Delta)) .
\end{array}
$$

Proof of Theorem 1. If $F \in \delta \tau_{\Delta}^{*}$ then a combination of Lemma 10 with $A=h(x)$, Lemma 11 with $a(x)=x-h(x)$, and Lemma 12 proves part (i) of Theorem 1.

To prove part (ii), assume that $F \in \mathcal{L}_{\Delta}$ and that the asymptotics in (3) holds. Then, from Lemma 10 with $a=0$ and Lemma 11 with $a(x)=x$, it follows that

$$
\int_{h(x)}^{x} H_{x}(\mathrm{~d} y) \mathrm{P}\{\tilde{M}+\xi \in x-y+\Delta, \xi \in x-y+\Delta\}=o(F(x+\Delta)) .
$$


Using the fact that $F \in \mathcal{L}_{\Delta}$ and Theorem 2, we have

$$
\begin{aligned}
& \int_{h(x)}^{x} H_{x}(\mathrm{~d} y) \mathrm{P}\{\tilde{M}+\xi \in x-y+\Delta, \xi \in x-y+\Delta\} \\
& \quad \geq \mathrm{P}\{M=0\} \int_{h(x)}^{x / 2} H_{x}(\mathrm{~d} y) F(x-y+\Delta) \\
& \quad \geq(1-o(1)) \mathrm{P}\{M=0\} \frac{\mathrm{E}(\tau)}{m} \int_{h(x)}^{x / 2} \mathrm{~d} y F((y, x]) F(x-y+\Delta) .
\end{aligned}
$$

Therefore,

$$
\int_{h(x)}^{x / 2} \mathrm{~d} y F((y, x]) F(x-y+\Delta)=o(F(x+\Delta)),
$$

which, according to Lemma 1 , implies that $F \in \delta \tau_{\Delta}^{*}$.

Lemma 11 describes the typical behaviour of the random walk resulting in the rare event $\left\{M_{\tau} \in x+\Delta\right\}$. For $a(x)=h(x)$, it shows that typically the random walk spends some time sufficiently close to 0 (in the interval $(0, h(x)]$ ), then makes a single jump to some point $z \in(x-h(x), x+T]$, and then, with probability $\pi(x-z+\Delta)$, reaches interval $x+\Delta$ from point $z$ and immediately goes down to $-\infty$. Note that the single big jump to the interval $(x, x+T]$ is only a part of this event.

Proof of Lemma 11. First we prove the upper bound. Clearly, for any set $B$,

$$
H_{a(x)}(B) \leq H_{\infty}(B)=\mathrm{E}(\tau) \pi(B)
$$

Thus,

$$
\begin{aligned}
\int_{0}^{h(x)} & H_{a(x)}(\mathrm{d} y) \mathrm{P}\{\tilde{M}+\xi \in x-y+\Delta, \xi \in(x-y-h(x), x-y+T]\} \\
& \leq \mathrm{E}(\tau) \int_{0}^{h(x)} \pi(\mathrm{d} y) \mathrm{P}\{\tilde{M}+\xi \in x-y+\Delta, \xi \in(x-y-h(x), x-y+T]\} \\
& \leq \mathrm{E}(\tau) \int_{0}^{h(x)} \pi(\mathrm{d} y) \mathrm{P}\{\tilde{M}+\xi \in x-y+\Delta, \tilde{M} \leq h(x)+T\} \\
& =\mathrm{E}(\tau) \int_{0}^{h(x)} \pi(\mathrm{d} y) \int_{0}^{h(x)+T} \pi(\mathrm{d} z) F(x-y-z+\Delta) \\
& \sim \mathrm{E}(\tau) F(x+\Delta),
\end{aligned}
$$

where the asymptotic equivalence follows from (14). This gives us the upper bound. To prove the lower bound, note that for any fixed $A$, uniformly in $u \in[0, A]$,

$$
H_{a(x)}([0, u]) \rightarrow \sum_{n=0}^{\infty} \mathrm{P}\left\{\tau>n, S_{n} \in[0, u]\right\}=\mathrm{E}(\tau) \pi([0, u]), \quad x \rightarrow \infty
$$


This convergence holds uniformly even on the interval $[0, g(x)]$, for some $g(x) \uparrow \infty$. Therefore,

$$
\begin{aligned}
& \int_{0}^{h(x)} \quad H_{a(x)}(\mathrm{d} y) \mathrm{P}\{\tilde{M}+\xi \in x-y+\Delta, \xi \in(x-y-h(x), x-y+T]\} \\
& \geq(1-o(1)) \mathrm{E}(\tau) \int_{0}^{g(x)} \pi(\mathrm{d} y) \mathrm{P}\{\tilde{M}+\xi \in x-y+\Delta, \\
& \quad \xi \in(x-y-h(x), x-y+T]\} \\
& \geq(1-o(1)) \mathrm{E}(\tau) \int_{0}^{g(x)} \pi(\mathrm{d} y) \mathrm{P}\{\tilde{M}+\xi \in x-y+\Delta, \tilde{M} \leq h(x)\} \\
& \sim \mathrm{E}(\tau) F(x+\Delta) .
\end{aligned}
$$

This gives us the lower bound.

Proof of Lemma 12. By Proposition 1 (see the appendix) and $F \in \mathcal{L}_{\Delta}$, for some constant $C_{1}>0$ and for $x$ and $y$ with $y \in(h(x), x-h(x))$, we have

$$
\begin{aligned}
\mathrm{P}\{\tilde{M} & +\xi \in x-y+\Delta, \xi \in(x-y-h(x), x-y+T]\} \\
\leq & \int_{0}^{h(x)+T} \pi(\mathrm{d} z) F(x-y-z+\Delta) \\
\leq & C_{1}\left(\int_{0}^{h(x)+T} \mathrm{~d} z F((z, x-y]) F(x-y-z+\Delta)\right. \\
& \left.\quad+\bar{F}(x-y) \int_{0}^{h(x)+T} \mathrm{~d} z F(x-y-z+\Delta)\right) \\
= & : C_{1}\left(P_{1}+P_{2}\right) .
\end{aligned}
$$

It follows from (8) that, for some constant $C_{2}$ and for $x$ and $y$ with $y \in(h(x), x-h(x))$,

$$
P_{1} \leq C_{2} F(x-y+\Delta)
$$

Furthermore,

$$
P_{2} \leq T \bar{F}(x-y) F((x-y-h(x)-T, x-y+T]) .
$$

We can start to estimate (15). Since $H_{x}(B)$ is monotonically increasing in $x$,

$$
\int_{h(x)}^{x-h(x)} H_{x-h(x)}(\mathrm{d} y) P_{1} \leq C_{1} C_{2} \int_{h(x)}^{x-h(x)} H_{x}(\mathrm{~d} y) F(x-y+\Delta) .
$$

By Theorem 2 and $F \in \mathcal{L}_{\Delta}$, the right-hand side is bounded from above as follows:

$$
\int_{h(x)}^{x-h(x)} H_{x}(\mathrm{~d} y) F(x-y+\Delta)=O(1) \int_{h(x)}^{x-h(x)} \mathrm{d} y F((y, x]) F(x-y+\Delta)=o(F(x+\Delta)) .
$$

Here the final equality follows from (6). 
Furthermore, Theorem 2 and $F \in \mathcal{L}_{\Delta}$ imply that

$$
\begin{aligned}
\int_{h(x)}^{x-}(x) & H_{x}(\mathrm{~d} y) P_{2} \\
\quad \leq & T \int_{h(x)}^{x-h(x)} H_{x}(\mathrm{~d} y) \bar{F}(x-y) F((x-y-h(x)-T, x-y+T]) \\
\quad \leq & O(1) \bar{F}(h(x)) \int_{h(x)}^{x-h(x)} \mathrm{d} y F((y, x]) F((x-h(x)-2 T-y, x+T-y]) \\
\quad= & O(1) \bar{F}(h(x)) F((x-h(x)-2 T, x+T]),
\end{aligned}
$$

where the equality follows from (10). Then

$$
\begin{aligned}
\bar{F}(h(x)) F((x-h(x)-2 T, x+T]) & \leq \bar{F}(h(x)) \sum_{n=-1}^{[h(x) / T]+2} F(x-n T+\Delta) \\
& \sim \frac{\bar{F}(h(x)) h(x) F(x+\Delta)}{T} \\
& =o(F(x+\Delta)),
\end{aligned}
$$

since $\bar{F}(x) x \rightarrow 0$ if $\int_{0}^{\infty} \bar{F}(u) \mathrm{d} u<\infty$. Here [·] denotes the integer-part function.

\section{Proof of Theorem 2}

In this section we prove Theorem 2, which gives the key estimates for the renewal function $H_{x}((y, y+b])$. We start with the lower bound.

Lemma 13. Let $F \in \mathcal{L}_{\Delta}$. Let $b>0$ be any number if $F$ is a nonlattice distribution, and let $b=n h, n \in \mathbb{N}$, if $F$ is a lattice distribution with step size $h$. Then, for any $\varepsilon>0$, there exists $a y_{0}>0$ such that, for $x$ and $y$ with $y \in\left(y_{0}, x-y_{0}\right)$,

$$
H_{x}((y, y+b]) \geq(1-\varepsilon) \mathrm{E}(\tau) \frac{b}{m} F((y, x]) .
$$

Proof. Let $\delta>0$ be such that $(1-\delta)^{4}=1-\varepsilon$. Let $y_{1}$ be a constant to be defined later. Let $\Delta_{b}=(0, b]$. Recall that $\mu\left(y_{1}\right)=\inf \left\{n \geq 1: S_{n}>y_{1}\right\}$. For $x>y+2 y_{1}$, by the Markov property we have

$$
\begin{aligned}
& H_{x}((y, y+b]) \geq \sum_{i=0}^{\infty} \mathrm{P}\left\{\mu\left(y_{1}\right) \leq \tau,\right. \\
& \quad S_{\mu\left(y_{1}\right)} \in\left(y+y_{1}, x-y_{1}\right], \\
&= \int_{y\left(y_{1}\right)+i}^{x-y_{1}} \mathrm{P}\left\{\mu\left(y_{1}\right) \leq \tau, S_{\mu\left(y_{1}\right)+i} \in y+S_{\mu\left(y_{1}\right)} \in \mathrm{d} u\right\} \\
& \times \sum_{i=0}^{\infty} \mathrm{P}\left\{M_{i} \leq x-u, S_{i} \in y-u+\Delta_{b}, \tau_{-u}>i\right\},
\end{aligned}
$$


where $\tau_{-u}=\min \left\{n \geq 1: S_{n}<-u\right\}$. Represent the infinite sum in the following form:

$$
\begin{aligned}
\sum_{i=0}^{\infty} \mathrm{P}\{ & \left.M_{i} \leq x-u, S_{i} \in x-u+\Delta_{b}, \tau_{-u}>i\right\} \\
& =E_{1}-E_{2} \\
& :=\sum_{i=0}^{\infty} \mathrm{P}\left\{M_{i} \leq x-u, S_{i} \in y-u+\Delta_{b}\right\} \\
& \quad-\sum_{i=0}^{\infty} \mathrm{P}\left\{M_{i} \leq x-u, S_{i} \in y-u+\Delta_{b}, \tau_{-u} \leq i\right\} .
\end{aligned}
$$

By the renewal theorem for random walks on the real line, for sufficiently large $y_{1}$ we have

$$
\begin{aligned}
E_{2} & \leq \sum_{i=0}^{\infty} \mathrm{P}\left\{S_{i} \in y-u+\Delta_{b}, \tau_{-u} \leq i\right\} \\
& =\int_{-\infty}^{-u} \mathrm{P}\left\{S_{\tau_{-u}} \in \mathrm{d} z\right\} \sum_{i=0}^{\infty} \mathrm{P}\left\{S_{i} \in y-u-z+\Delta_{b}\right\} \\
& \leq \sup _{v>y} \sum_{i=0}^{\infty} \mathrm{P}\left\{S_{i} \in v+\Delta_{b}\right\} \\
& \leq \sup _{v>2 y_{1}} \sum_{i=0}^{\infty} \mathrm{P}\left\{S_{i} \in v+\Delta_{b}\right\} \\
& \leq \delta \frac{b}{3 m} .
\end{aligned}
$$

Furthermore,

$$
\begin{aligned}
E_{1} & =\sum_{i=0}^{\infty} \mathrm{P}\left\{S_{i} \in y-u+\Delta_{b}\right\}-\sum_{i=0}^{\infty} \mathrm{P}\left\{M_{i}>x-u, S_{i} \in y-u+\Delta_{b}\right\} \\
& =: E_{11}-E_{12} .
\end{aligned}
$$

By the Markov property and the renewal theorem, for some $C>0$ we have

$$
E_{12} \leq \int_{x-u}^{\infty} \mathrm{P}\left\{S_{\mu(x-u)} \in \mathrm{d} z\right\} \sum_{i=0}^{\infty} \mathrm{P}\left\{S_{i} \in y-u-z+\Delta_{b}\right\} \leq C \mathrm{P}\{\mu(x-u)<\infty\} \leq \delta \frac{b}{3 m}
$$

The latter inequality holds for sufficiently large $y_{1}$, since $x-u>y_{1}$ and $\mathrm{P}\left\{\mu\left(y_{1}\right)<\infty\right\} \rightarrow 0$ as $y_{1} \rightarrow \infty$. Again by the renewal theorem, for sufficiently large $y_{1}$ we have

$$
E_{11} \geq \inf _{u \in\left(y+y_{1}, x-y_{1}\right)} \sum_{i=0}^{\infty} \mathrm{P}\left\{S_{i} \in y-u+\Delta_{b}\right\} \geq \inf _{v<-y_{1}} \sum_{i=0}^{\infty} \mathrm{P}\left\{S_{i} \in v+\Delta_{b}\right\} \geq\left(1-\frac{\delta}{3}\right) \frac{b}{m}
$$


Fix a positive integer $N$. We can write

$$
\begin{aligned}
H_{x}((y, y+b]) & \geq(1-\delta) \frac{b}{m} \mathrm{P}\left\{\mu\left(y_{1}\right) \leq \tau, S_{\mu\left(y_{1}\right)} \in\left(y+y_{1}, x-y_{1}\right]\right\} \\
& \geq(1-\delta) \frac{b}{m} \sum_{n=0}^{N} \int_{0}^{y_{1}} \mathrm{P}\left\{\tau>n, M_{n} \leq y_{1}, S_{n} \in \mathrm{d} t\right\} \int_{y-t+y_{1}}^{x-t-y_{1}} F(\mathrm{~d} z) \\
& \geq(1-\delta) \frac{b}{m} \sum_{n=0}^{N} \mathrm{P}\left\{\tau>n, M_{n} \leq y_{1}\right\} \inf _{0<t<y_{1}} F\left(\left(y-t+y_{1}, x-t-y_{1}\right]\right) .
\end{aligned}
$$

By now taking $N$ and $y_{1}$ to be sufficiently large, we can estimate the sum from below:

$$
\sum_{n=0}^{N} \mathrm{P}\left\{\tau>n, M_{n} \leq y_{1}\right\} \geq(1-\delta) \sum_{n=0}^{N} \mathrm{P}\{\tau>n\} \geq(1-\delta)^{2} \mathrm{E}(\tau)
$$

Finally, by Lemma 4 , we can choose a $y_{0}>y_{1}$ such that

$$
\inf _{0<t<y_{1}} F\left(\left(y-t+y_{1}, x-t-y_{1}\right]\right) \geq F\left(\left(y+y_{1}, x-2 y_{1}\right]\right) \geq(1-\delta) F((y, x]) .
$$

The upper bound, as usual, is more difficult to analyse. Let $\mathrm{P}\{M=0\}=p$ and $q=1-p$. It is known that $\mathrm{E}(\tau)=1 / p$; see, e.g. [2, Chapter VIII, Theorem 2.3]. We will use a different representation for $H_{x}(B)$. For any measurable set $B$, by duality of random walks (see [10, Section XII.2]),

$$
\begin{aligned}
H_{x}(B) & =\mathbf{1}_{\{0 \in B\}}+\sum_{n=1}^{\infty} \mathrm{P}\left\{S_{1}>0, S_{2}>0, \ldots, S_{n}>0, M_{n} \leq x, S_{n} \in B\right\} \\
& =\mathbf{1}_{\{0 \in B\}}+\sum_{n=1}^{\infty} \mathrm{P}\left\{S_{n}>M_{n-1}, S_{n}-\min _{i=0, \ldots, n-1} S_{i} \leq x, S_{n} \in B\right\}
\end{aligned}
$$

Let $v_{0}, v_{1}, v_{2}, \ldots$ be the sequence of (strictly) increasing ladder epochs:

$$
\begin{aligned}
v_{0}=0, \quad v_{1}=\inf \left\{n \geq 1: S_{n}\right. & >0\} \leq \infty, \quad \ldots, \\
v_{k} & =\inf \left\{n \geq 1: S_{n}>S_{v_{k-1}}\right\} \leq \infty,
\end{aligned}
$$

Then (16) can be written as

$$
H_{x}(B)=\sum_{k=0}^{\infty} G_{x}^{(k)}(B):=\sum_{k=0}^{\infty} \mathrm{P}\left\{S_{v_{k}} \in B, S_{v_{k}} \leq x+\min _{i=0, \ldots, v_{k}} S_{i}\right\}
$$

Here $G_{x}^{(0)}(B)=\mathbf{1}_{\{0 \in B\}}$. Thus, $\mathrm{P}\left\{v_{1}<\infty\right\}=\mathrm{P}\{M>0\}=q<1$ and, therefore,

$$
G_{x}^{(k)}((0, \infty)) \leq q^{k}, \quad k \geq 1
$$


To estimate $H_{x}((y, y+b])$ from above, we will recursively construct estimates for $G_{x}^{(k)}((y, y+b])$. Let

$$
G_{x}(B)=\mathrm{P}\left\{-S_{v_{1}-1} \leq x, S_{v_{1}} \in B\right\} .
$$

Note that, for any $x, G_{x}((0, \infty)) \leq q<1$. For $k=1$, we have

$$
\begin{aligned}
G_{x}^{(1)}((y, y+b]) & \leq \mathrm{P}\left\{S_{v_{1}} \in(y, y+b],-S_{\nu_{1}-1} \leq x-S_{\nu_{1}}\right\} \\
& \leq \mathrm{P}\left\{S_{\nu_{1}} \in(y, y+b],-S_{\nu_{1}-1} \leq x-y\right\} \\
& =G_{x-y}(y+\Delta) .
\end{aligned}
$$

Again let $\Delta_{b}=(0, b]$. For $k>1$, by the Markov property we have

$$
\begin{aligned}
& G_{x}^{(k)}\left(y+\Delta_{b}\right) \\
& =\mathrm{P}\left\{S_{v_{k}} \in y+\Delta_{b}, S_{v_{k}} \leq x+\min _{i=0, \ldots, v_{k}} S_{i},-S_{v_{1}-1} \leq x-S_{v_{k}}, S_{v_{1}}<S_{v_{k}}\right\} \\
& \leq \mathrm{P}\left\{S_{v_{k}} \in y+\Delta_{b}, S_{v_{k}} \leq x+\min _{i=v_{1}, \nu_{1}+1, \ldots, \nu_{k}} S_{i},-S_{v_{1}-1} \leq x-y, S_{v_{1}} \leq y+b\right\} \\
& =\int_{0}^{y+b} \mathrm{P}\left\{-S_{\nu_{1}-1} \leq x-y, S_{\nu_{1}} \in \mathrm{d} t\right\} \mathrm{P}\left\{S_{\nu_{k}}-S_{\nu_{1}} \in(y-t, y-t+b]\right. \text {, } \\
& \left.S_{v_{k}}-S_{\nu_{1}} \leq x+\min _{i=v_{1}, v_{1}+1, \ldots, v_{k}} S_{i}-S_{v_{1}}\right\} \\
& =\int_{0}^{y+b} G_{x-y}(\mathrm{~d} t) G_{x}^{(k-1)}\left(y-t+\Delta_{b}\right) .
\end{aligned}
$$

Before starting to estimate $G_{x}^{(k)}$, we need to prove two auxiliary lemmas.

Lemma 14. Let $F \in \mathcal{L}_{\Delta}$. Let $b>0$ be any number if $F$ is a nonlattice distribution, and let $b=n h, n \in \mathbb{N}$, if $F$ is a lattice distribution with step size $h$. For any $\varepsilon>0$, there exists some $z_{0}$ such that, for any $x$ and $z$ with $x>z>z_{0}$,

$$
G_{x}((z, z+b]) \leq(1+\varepsilon) \frac{p}{m} b F((z, x+z]) .
$$

Also, there exists a constant $H_{-}(b)$ such that, for any $x, z>0$,

$$
G_{x}((z, z+b]) \leq H_{-}(b) F((z, x+z+b]) .
$$

Proof. Clearly,

$$
\begin{aligned}
& G_{x}((z, z+b]) \\
& \quad=\sum_{n=1}^{\infty} \mathrm{P}\left\{S_{1} \leq 0, \ldots, S_{n-1} \leq 0, S_{n}>0,-S_{n-1} \leq x, S_{n-1}+\xi_{n} \in(z, z+b]\right\} \\
& \quad=\int_{z}^{x+z+b} F(\mathrm{~d} t) \sum_{n=1}^{\infty} \mathrm{P}\left\{S_{1} \leq 0, \ldots, S_{n-1} \leq 0,-S_{n-1} \in[t-z-b, t-z)\right\} \\
& \quad=\int_{z}^{x+z+b} F(\mathrm{~d} t) H_{-}([t-z-b, t-z)) .
\end{aligned}
$$


Here, for any measurable set $B, H_{-}(B)=\sum_{k=0}^{\infty} G_{-}^{* k}(B)$, where $G_{-}$is the distribution function of the decreasing ladder height; see, e.g. [2, Chapter VIII, Theorem 2.3]. Thus, (20) follows from the subadditivity of the renewal measure, i.e. $H_{-}([t-z-b, t-z)) \leq H_{-}([0, b]) \equiv H_{-}(b)$. Furthermore, by the renewal theorem, $H_{-}([u-b, u)) \rightarrow \int_{0}^{\infty} G_{-}(\mathrm{d} t) t=(p / m) b$. Thus, for sufficiently large $z_{1}$ and $x>z_{1}$,

$$
G_{x}((z, z+b]) \leq H_{-}(b) F\left(\left(z, z+z_{1}\right]\right)+\left(1+\frac{\varepsilon}{2}\right) \frac{p}{m} b F\left(\left(z+z_{1}, x+z+b\right]\right) .
$$

For any $\delta>0$, it follows from Lemma 4 that there exists a $z_{0} \equiv z_{0}(\delta)$ such that, for $x$ and $z$ with $x+z-z_{0}>z>z_{0}$,

$$
F\left(\left(z, z+z_{1}\right]\right) \leq \delta F((z, x]), \quad F\left(\left(z+z_{1}, x+z+b\right]\right) \leq(1+\delta) F((z, x]) .
$$

Then, by taking $\delta$ sufficiently small we immediately obtain our assertion from (21).

Lemma 15. Let $F \in \delta \tau_{\Delta}^{*}$ and let $b>0$ be a constant. For any $\varepsilon>0$ there exists $a y_{0}>0$ such that, for $x$ and $y$ with $y \in\left(y_{0}, x-y_{0}\right)$,

$$
\int_{0}^{y-y_{0}} G_{x-y}(\mathrm{~d} t) F((y-t, x+b]) \leq q(1+\varepsilon) F((y, x]) .
$$

Proof. Since $F \in \delta \tau_{\Delta}^{*}$, by Lemmas 4 and 5 there exists a constant $x_{1}>0$ such that, for $x$ and $y$ with $y \in\left(x_{1}, x-x_{1}\right)$,

$$
\begin{gathered}
\sup _{y>x_{1}-1, y<x-x_{1}+1} \frac{F((y-2, x+b+2])}{F((y, x])} \leq 2, \\
\int_{x_{1}-1}^{y-x_{1}+1} F((y-t, x-t]) F((t, x]) \mathrm{d} t \leq q \frac{\varepsilon}{4 H_{-}(1)} .
\end{gathered}
$$

Furthermore, by Lemma 4 there exists a $y_{0}>x_{1}$ such that, for $x$ and $y$ with $y \in\left(y_{0}, x-y_{0}\right)$,

$$
F\left(\left(y-x_{1}, x+b\right]\right) \leq\left(1+\frac{\varepsilon}{2}\right) F((y, x]) .
$$

Splitting integral (22) into two yields

$$
\begin{aligned}
\int_{0}^{y-x_{1}} & G_{x-y}(\mathrm{~d} t) F((y-t, x+T]) \\
= & I_{1}+I_{2} \\
:= & \int_{0}^{x_{1}} G_{x-y}(\mathrm{~d} t) F((y-t, x+T])+\int_{x_{1}}^{y-x_{1}} G_{x-y}(\mathrm{~d} t) F((y-t, x+T]) .
\end{aligned}
$$

First, it follows from (25) that, for $x$ and $y$ with $y \in\left(y_{0}, x-y_{0}\right)$,

$$
I_{1} \leq F\left(\left(y-x_{1}, x+b\right]\right) \int_{0}^{x_{1}} G_{x-y}(\mathrm{~d} t) \leq q F\left(\left(y-x_{1}, x+T\right]\right) \leq q\left(1+\frac{\varepsilon}{2}\right) F((y, x]) .
$$


Second, by Lemma 14,

$$
\begin{aligned}
I_{2} & \leq \sum_{n=\left[x_{1}\right]}^{\left[y-x_{1}\right]} G_{x-y}((n, n+1]) F((y-n-1, x+b]) \\
& \leq H_{-}(1) \sum_{n=\left[x_{1}\right]}^{\left[y-x_{1}\right]} F((n, n+x-y+1]) F((y-n-1, x+b]) \mathrm{d} u \\
& \leq H_{-}(1) \int_{x_{1}-1}^{y-x_{1}+1} F((u-1, u+x-y+2]) F((y-u-2, x+b]) \mathrm{d} u \\
& =H_{-}(1) \int_{x_{1}-1}^{y-x_{1}+1} F((y-t-1, x-t+2]) F((t-2, x+b]) \mathrm{d} t .
\end{aligned}
$$

By now applying (23) and (24), we obtain

$$
I_{2} \leq 2 H_{-}(1) \int_{x_{1}-1}^{y-x_{1}+1} F((y-t, x-t]) F((t, x]) \mathrm{d} t \leq q \frac{\varepsilon}{2} F((y, x]) .
$$

Lemma 16. Let $F \in \delta \tau_{\Delta}^{*}$. For any $\varepsilon>0$, there exists an increasing sequence $\left\{y_{k}>0\right\}$ such that, for $x$ and $y$ with $y \in\left(y_{k}, x-y_{k}\right)$,

$$
G_{x}^{(k)}((y, y+b]) \leq(1+\varepsilon)^{k} k p q^{k-1} \frac{b}{m} F((y, x]) .
$$

Proof. We will give a proof by induction. For $k=1$, by Lemma 14 and (18) there exists some $y_{1}$ such that, for $x$ and $y$ with $y \in\left(y_{1}, x-y_{1}\right)$,

$$
G_{x}^{(1)}((y, y+b]) \leq G_{x-y}((y, y+b]) \leq(1+\varepsilon) \frac{p b}{m} F((y, x]) .
$$

We now suppose that we have proved our assertion for some $k-1$, and prove it for $k$. By (19), for $x$ and $y$ with $y \in\left(y_{k-1}, x-y_{k-1}\right)$,

$$
\begin{aligned}
G_{x}^{(k)}( & \left.y+\Delta_{b}\right) \\
& \leq \int_{0}^{y+b} G_{x-y}(\mathrm{~d} t) G_{x}^{(k-1)}\left(y-t+\Delta_{b}\right) \\
& =\int_{0}^{y-y_{k-1}} G_{x-y}(\mathrm{~d} t) G_{x}^{(k-1)}\left(y-t+\Delta_{b}\right)+\int_{y-y_{k-1}}^{y+b} G_{x-y}(\mathrm{~d} t) G_{x}^{(k-1)}\left(y-t+\Delta_{b}\right) \\
& =: P_{1}+P_{2} .
\end{aligned}
$$

By the induction hypothesis and Lemma 15, for some sufficiently large $y_{k} \geq 3 y_{k-1}$ and $x$ and $y$ with $y \in\left(y_{k}, x-y_{k}\right)$,

$$
\begin{aligned}
P_{1} & =\int_{0}^{y-y_{k-1}} G_{x-y}(\mathrm{~d} t) G_{x}^{(k-1)}\left(y-t+\Delta_{b}\right) \\
& \leq(1+\varepsilon)^{k-1}(k-1) p q^{k-2} \frac{b}{m} \int_{0}^{y-y_{k-1}} G_{x-y}(\mathrm{~d} t) F((y-t, x]) \\
& \leq(1+\varepsilon)^{k}(k-1) p q^{k-1} \frac{b}{m} F((y, x])
\end{aligned}
$$


By integrating $P_{2}$ by parts and applying Lemma 14 for $x$ and $y$ with $y \in\left(y_{k}, x-y_{k}\right)$, we obtain

$$
\begin{aligned}
P_{2} & =\int_{0}^{y_{k-1}+b} \mathrm{~d} G_{x}^{(k-1)}(z) G_{x-y}\left(y-z+\Delta_{b}\right) \\
& \leq \frac{p b}{m}(1+\varepsilon) \int_{0}^{y_{k-1}+b} \mathrm{~d} G_{x}^{(k-1)}(z) F((y-z, x-z+b]) \\
& \leq \frac{p b}{m}(1+\varepsilon) q^{k-1} \sup _{0 \leq z \leq y_{k-1}+b} F((y-z, x-z+b]) \\
& \leq \frac{p b}{m}(1+\varepsilon)^{2} q^{k-1} F((y, x]),
\end{aligned}
$$

where $G_{x}^{(k-1)}(z) \equiv G_{x}^{(k-1)}((0, z])$. The final inequality follows from Lemma 4, since we can increase $y_{k}$ again if necessary.

Lemma 17. Let $F \in \delta \tau_{\Delta}^{*}$. For any $\varepsilon>0$, there exist $y_{0}>0$ and $C>0$ such that, for $x$ and $y$ with $y \in\left(y_{0}, x-y_{0}\right)$, and for any $k \geq 1$,

$$
G_{x}^{(k)}((y, y+b]) \leq C q^{k}(1+\varepsilon)^{k} F((y, x]) .
$$

Proof. The proof is quite similar to the proof of the exponential bound for local subexponential distributions (see [3, Proposition 4]). Let $y_{0}$ be such that Lemma 15 holds with $\varepsilon$ replaced by $\varepsilon / 2$, and let

$$
A_{k}:=\sup _{\left\{x, y: y_{0}<y<x-y_{0}\right\}} \frac{G_{x}^{(k)}\left(y+\Delta_{b}\right)}{F((y, x])} .
$$

It follows from Lemma 14 that $A_{1}$ is finite. For any $k>1$ and $y>y_{0}$, by (19) we have

$$
\begin{aligned}
G_{x}^{(k)}( & \left.y+\Delta_{b}\right) \\
& \leq \int_{0}^{y+b} G_{x-y}(\mathrm{~d} t) G_{x}^{(k-1)}\left(y-t+\Delta_{b}\right) \\
& =\int_{0}^{y-y_{0}} G_{x-y}(\mathrm{~d} t) G_{x}^{(k-1)}\left(y-t+\Delta_{b}\right)+\int_{y-y_{0}}^{y+b} G_{x-y}(\mathrm{~d} t) G_{x}^{(k-1)}\left(y-t+\Delta_{b}\right) \\
& =: P_{1}+P_{2} .
\end{aligned}
$$

By the definition of $A_{k-1}$ and Lemma 15 , for $y<x-y_{0}$ we have

$$
\begin{aligned}
P_{1} & =\int_{0}^{y-y_{0}} G_{x-y}(\mathrm{~d} t) G_{x}^{(k-1)}\left(y-t+\Delta_{b}\right) \\
& \leq A_{k-1} \int_{0}^{y-y_{0}} G_{x-y}(\mathrm{~d} t) F((y-t, x]) \\
& \leq A_{k-1} q\left(1+\frac{\varepsilon}{2}\right) F((y, x]) .
\end{aligned}
$$


Integrating $P_{2}$ by parts and applying Lemma 14 yields

$$
\begin{aligned}
P_{2} & =\int_{0}^{y_{0}+b} \mathrm{~d} G_{x}^{(k-1)}(z) G_{x-y}\left(y-z+\Delta_{b}\right) \\
& \leq H_{-}(b) \int_{0}^{y_{0}+b} \mathrm{~d} G_{x}^{(k-1)}(z) F((y-z, x-z+b]) \\
& \leq H_{-}(b) q^{k-1} \sup _{0 \leq z \leq y_{0}+b} F((y-z, x-z+b]) .
\end{aligned}
$$

Then, by (17), for $y>2 y_{0}$ we have

$$
P_{2} \leq q^{k-1} \sup _{0 \leq z<y_{0}+T} F((y-z, x-z+T]) \leq q^{k-1} L_{1} F((y, x]),
$$

where

$$
L_{1}:=\sup _{y<x-y_{0}, y>2 y_{0}, 0 \leq z<y_{0}+T} \frac{F((y-z, x-z+T])}{F((y, x])}<\infty .
$$

If $y_{0}<y \leq 2 y_{0}$ then, by (17),

$$
\frac{P_{2}}{F((y, x])} \leq \frac{q^{k-1}}{\inf _{y_{0}<y \leq 2 y_{0}, x>y-y_{0}} F((y, x])} \leq \frac{q^{k-1}}{\inf _{y_{0}<y \leq 2 y_{0}} F((y, y+T])}=: L_{2} .
$$

Let $R=H_{-}(b) L_{1}+L_{2}$. Then, for any $y>y_{0}$,

$$
A_{k} \leq q\left(1+\frac{\varepsilon}{2}\right) A_{k-1}+q^{k-1} R .
$$

Therefore, by induction, for any $k$ we have

$$
A_{k} \leq A_{1} q^{k-1}\left(1+\frac{\varepsilon}{2}\right)^{k-1}+R \sum_{l=0}^{k-2} q^{k-1-l} q^{l}\left(1+\frac{\varepsilon}{2}\right)^{l} \leq \max \left(A_{1}, R\right) k q^{k-1}\left(1+\frac{\varepsilon}{2}\right)^{k-1}
$$

Thus, the assertion of the lemma holds with

$$
C=\max \left(A_{1}, R\right) \sup _{k \geq 1} \frac{k(1+\varepsilon / 2)^{k-1}}{q(1+\varepsilon)^{k}}<\infty .
$$

Proof of Theorem 2. The lower bound was proved in Lemma 13. To prove the upper bound, fix a $\delta>0$. By Lemma 17, there exist some $C \equiv C(\delta)$ and $\tilde{y}_{0}$ such that exponential bound (27) holds for $x$ and $y$ with $y \in\left(\tilde{y}_{0}, x-\tilde{y}_{0}\right)$. Let $\left\{y_{k}\right\}$ be the sequence constructed in Lemma 16, for this $\delta$. Without loss of generality, we may assume that $y_{k} \geq \tilde{y}_{0}$ for all $k$. Fix some $n \in \mathbb{N}$. Then, for $x$ and $y$ with $y \in\left(y_{n}, x-y_{n}\right)$,

$$
\begin{aligned}
H_{x}((y, y+b]) & =\sum_{k=0}^{n} G_{x}^{(k)}((y, y+b])+\sum_{k=n+1}^{\infty} G_{x}^{(k)}((y, y+b]) \\
& \leq F((y, x])\left(\sum_{k=1}^{n}(1+\delta)^{k} k p q^{k-1} \frac{b}{m}+C \sum_{k=n+1}^{\infty} q^{k}(1+\delta)^{k}\right) \\
& \leq F((y, x])\left(\frac{p b}{m} \frac{1+\delta}{(1-q-q \delta)^{2}}+C \frac{q^{n+1}(1+\delta)^{n+1}}{1-q-q \delta}\right) .
\end{aligned}
$$


Let $\delta$ and $n$ be such that

$$
\frac{1+\delta}{(1-q-q \delta)^{2}} \leq \frac{1+\varepsilon / 2}{(1-q)^{2}}, \quad C \frac{q^{n+1}(1+\delta)^{n+1}}{1-q-q \delta} \leq \frac{p b}{m} \frac{\varepsilon / 2}{(1-q)^{2}} .
$$

Then we can let $y_{0}=y_{n}$ and, for $x$ and $y$ with $y \in\left(y_{0}, x-y_{0}\right)$, have

$$
H_{x}((y, y+b]) \leq(1+\varepsilon) \frac{b}{m} \frac{p}{(1-q)^{2}} F((y, x])=(1+\varepsilon) \frac{b}{m} \mathrm{E}(\tau) F((y, x]),
$$

since $q=1-p$ and $\mathrm{E}(\tau)=1 / p$.

\section{Example}

Example 1. The aim of this example is to show that for any $F \in \mathcal{L}_{\Delta}$ there exists a stopping time such that

$$
\limsup \frac{\mathrm{P}\left\{M_{\sigma} \in x+\Delta\right\}}{F(x+\Delta)}>\mathrm{E}(\sigma) .
$$

This implies that the asymptotics $\mathrm{P}\left\{M_{\sigma}>x\right\} \sim \mathrm{E}(\sigma) F(x+\Delta)$ does not hold.

For each positive integer $n$, let $x_{n}=n^{2}$ and

$$
A=(-\infty, 0] \cup \bigcup_{n=0}^{\infty}\left(x_{n}, x_{n}+n\right] .
$$

Define the stopping time $\sigma=\inf \left\{n \geq 1: S_{n} \in A\right\}$. It is clear that $\sigma \leq \tau$ and, therefore, that $\mathrm{E}(\sigma)<\infty$. Note that, for $n>T$,

$$
\left\{M_{\sigma} \in x_{n}+\Delta\right\}=\left\{S_{\sigma} \in x_{n}+\Delta\right\},
$$

since if the random walk reaches an interval $x_{n}+\Delta$ it immediately stops.

Let $h(x) \uparrow \infty$, with $h(x) \leq x / 2$ being a function such that $F(x-y+\Delta) \sim F(x+\Delta)$ uniformly in $y \leq h(x)$. Fix a positive integer $N$. For some positive constant $K>T$, which we define below, and for $n>K+1$, we have

$$
\begin{aligned}
\mathrm{P}\left\{M_{\sigma} \in x_{n}+\Delta\right\} \geq & \sum_{i=0}^{N} \mathrm{P}\left\{\sigma>i, M_{i} \leq h\left(x_{n}\right), S_{i+1} \in x_{n}+\Delta\right\} \\
& +\mathrm{P}\left\{\xi_{1} \in x_{n}-A+\Delta, \xi_{1}+\xi_{2} \in x_{n}+\Delta\right\} \\
= & : P_{1}\left(x_{n}\right)+P_{2}\left(x_{n}\right) .
\end{aligned}
$$

For the first term we have

$$
\begin{aligned}
P_{1}\left(x_{n}\right) & =\sum_{i=0}^{N} \int_{0}^{h\left(x_{n}\right)} \mathrm{P}\left\{\sigma>i, M_{i} \leq h\left(x_{n}\right), S_{i} \in \mathrm{d} y, S_{i+1} \in x_{n}+\Delta\right\} \\
& \geq \sum_{i=0}^{N} \mathrm{P}\left\{\sigma>i, M_{i} \leq h\left(x_{n}\right)\right\} \inf _{y \leq h\left(x_{n}\right)} F\left(x_{n}-y+\Delta\right) \\
& =(1+o(1)) \sum_{i=0}^{N} \mathrm{P}\{\sigma>i\} F\left(x_{n}+\Delta\right), \quad n \rightarrow \infty .
\end{aligned}
$$


Letting $N \rightarrow \infty$, we obtain

$$
P_{1}\left(x_{n}\right) \geq(1-o(1)) \mathrm{E}(\sigma) F\left(x_{n}+\Delta\right), \quad n \rightarrow \infty .
$$

For the second term we have

$$
\begin{aligned}
P_{2}\left(x_{n}\right) & =\int_{x_{n}-K}^{x_{n}-K+T} F(\mathrm{~d} y) F\left(x_{n}-y+\Delta\right) \\
& \geq F\left(x_{n}-A+\Delta\right) \inf _{y \in x_{n}-A+\Delta} F\left(x_{n}-y+\Delta\right) \\
& =F\left(x_{n}-A+\Delta\right) \inf _{x \in[A-T, A)} F(z+\Delta) .
\end{aligned}
$$

Now let $K$ be such that $C=\inf _{x \in[K-T, K)} F(z+\Delta)>0$. Such a $K$ always exists since $F \in \mathscr{L}_{\Delta}$. Then

$$
P_{2}\left(x_{n}\right) \geq(1+o(1)) C F\left(x_{n}+\Delta\right), \quad n \rightarrow \infty .
$$

Therefore, strict inequality (28) holds.

\section{Appendix A.}

\section{A.1. Estimate for the local probabilities $\pi((x, x+b])$}

In our work we need the following proposition (see [4, Theorem 1]).

Proposition 1. Let $\mathrm{E}(\xi)=-m \in(-\infty, 0)$ and let $F \in 8^{*}$. There exist constants $c_{1}$ and $c_{2}$ such that, for $x, b \geq 0$,

$$
\pi((x, x+b]) \leq\left(c_{1}+c_{2} b\right) \bar{F}(x) .
$$

Remark 5. We actually need this estimate only for a fixed value of $b$. Then, under the additional assumption that $F \in \delta \tau_{\Delta}^{*}$ (which is sufficient for our purposes), the estimate follows from Theorem 2. Indeed, if we let $x=\infty$ in Theorem 2, then $H_{\infty}((y, y+b]) \leq C \bar{F}(y)$ for some $C$ and all $y$. Proposition 1 then follows from the fact that $H_{\infty}((y, y+b])=\mathrm{E}(\tau) \pi((x, x+b])$.

\section{A.2. Proofs}

Here we present the proofs of the lemmas stated in Section 2. Throughout the appendix, $h(x)$ denotes a function such that $h(x) \uparrow \infty$ as $x \rightarrow \infty, h(x)<x / 2$ for all $x$, and (2) holds uniformly in $|t| \leq h(x)$.

Proof of Lemma 1. First, for such an $h(x)$,

$$
\int_{0}^{h(x)} F((y, x]) F(x-y+\Delta) \sim m_{+} F(x+\Delta) .
$$

Thus, equivalence (5) follows directly from the definition of $\delta \tau_{\Delta}^{*}$. To prove (6) we need only show that if $F \in \delta \tau_{\Delta}^{*}$ then

$$
\int_{x / 2}^{x-h(x)} F((y, x]) F(x-y+\Delta) \mathrm{d} y=o(F(x+\Delta)) .
$$


We have

$$
\begin{aligned}
0 & \leq \int_{x / 2}^{x-h(x)} F((y, x]) F(x-y+\Delta) \mathrm{d} y \\
& =\int_{h(x)}^{x / 2} F((x-y, x])(F((y, x])-F((y+T, x])) \mathrm{d} y \\
& =\int_{h(x)}^{x / 2} F((x-y, x]) F((y, x]) \mathrm{d} y-\int_{h(x)+T}^{x / 2+T} F((x-y+T, x]) F((y, x]) \mathrm{d} y \\
& \leq \int_{h(x)+T}^{x / 2} F(x-y+\Delta) F((y, x]) \mathrm{d} y+\int_{h(x)}^{h(x)+T} F((x-y, x]) F((y, x]) \mathrm{d} y \\
& =: I_{1}+I_{2} .
\end{aligned}
$$

By (5), $I_{1}=o(F(x+\Delta))$. Furthermore,

$$
\begin{aligned}
I_{2} & \leq T \bar{F}(h(x)) F((x-h(x)-T, x]) \\
& \leq T \bar{F}(h(x)) \sum_{k=-1}^{[h(x)]} F(x-h(x)+k T+\Delta) \\
& =(1+o(1)) T \bar{F}(h(x))([h(x)]+2) F(x+\Delta) \\
& =o(F(x+\Delta)),
\end{aligned}
$$

since $x \bar{F}(x) \rightarrow 0$ if $\mathrm{E}(\xi)<\infty$. Equivalence (7) follows immediately from (6) and (29). To prove (8), arguing as above yields

$$
\begin{array}{rl}
\int_{x / 2}^{x} & F(x-y+\Delta) F((y, x]) \mathrm{d} y \\
& \leq \int_{T}^{x / 2} F(x-y+\Delta) F((y, x]) \mathrm{d} y+\int_{0}^{T} F((x-y, x]) F((y, x]) \mathrm{d} y \\
& \leq(1+o(1)) m_{+} F(x+\Delta)+F((x-T, x]) m_{+} \\
& \leq 2 m_{+}(1+o(1)) F(x+\Delta) .
\end{array}
$$

Proof of Lemma 2. For $x>x_{0}$, we have

$\int_{h(x)}^{x / 2} F(x-y+\Delta) F((y, x]) \mathrm{d} y \leq \frac{1}{c} F(x+\Delta) \int_{h(x)}^{x / 2} \bar{F}(y) \mathrm{d} y=o(F(x+\Delta)) \quad$ as $x \rightarrow \infty$.

Then $F \in \delta \tau_{\Delta}^{*}$ by Lemma 1 .

Proof of Lemma 3. By Lemma 1, it is sufficient to prove that

$$
\int_{h(x)}^{x / 2} \frac{F(x-y+\Delta)}{F(x+\Delta)} F((y, x]) \mathrm{d} y \leq \int_{h(x)}^{x / 2} \frac{F(x-y+\Delta)}{F(x+\Delta)} \bar{F}(y) \mathrm{d} y \rightarrow 0 \quad \text { as } x \rightarrow \infty,
$$

for the function $h(x)$. Consider the integrand:

$$
\begin{aligned}
\frac{F(x-y+\Delta)}{F(x+\Delta)} \bar{F}(y) & =\exp \left\{Q_{\Delta}(x)-Q_{\Delta}(x-y)-Q(y)\right\} \\
& \leq \exp \left\{y(r+\varepsilon) \frac{Q(x-y)}{x-y}-Q(y)\right\}
\end{aligned}
$$


for sufficiently large $x$. Since the function $Q(x) / x$ is eventually nonincreasing and $y \leq x / 2$,

$$
\exp \left\{y(r+\varepsilon) \frac{Q(x-y)}{x-y}-Q(y)\right\} \leq \exp \{-(1-r-\varepsilon) Q(y)\}=\bar{F}^{1-r-\varepsilon}(y)
$$

and the result follows.

Proof of Lemma 4. Clearly, for $y_{0}>3 t / \varepsilon+2 T$,

$$
\begin{aligned}
\frac{F((y-t, y])}{F((y, x])} & \leq \frac{F((y-t, y])}{F((y, y+3 t / \varepsilon+2 T])} \\
& \leq \frac{\sum_{n=0}^{[t]} F(y-t+n T+\Delta)}{\sum_{n=0}^{[3 t / \varepsilon]+1} F(y+n T+\Delta)} \\
& \leq \frac{4}{3} \frac{\sum_{n=0}^{[t]} F(y+\Delta)}{\sum_{n=0}^{[3 t / \varepsilon]+1} F(y+\Delta)} \\
& \leq \frac{4}{9} \varepsilon,
\end{aligned}
$$

where the third inequality holds for sufficiently large $y_{0}$ since $F \in \mathcal{L}_{\Delta}$. Similarly, it can be shown that, for sufficiently large $y_{0}, F((x, x+T]) \leq \frac{5}{9} \varepsilon F((y, x])$.

Proof of Lemma 5. Clearly

$$
\begin{aligned}
\int_{y_{0}}^{y-y_{0}} F((y-u, x-u]) F((u, x]) \mathrm{d} u= & \int_{y_{0}}^{y-y_{0}} F((y-u, x-u]) F((u, y]) \mathrm{d} u \\
& +\int_{y_{0}}^{y-y_{0}} F((y-u, x-u]) F((y, x]) \mathrm{d} u \\
= & : I_{1}+I_{2} .
\end{aligned}
$$

First, by Lemma 1, for sufficiently large $y_{0}$ we have

$$
\begin{aligned}
I_{1} & \leq \sum_{n=0}^{[(x-y) / T]} \int_{y_{0}}^{y-y_{0}} F(y+n T-u+\Delta) F((u, y]) \mathrm{d} u \\
& \leq \sum_{n=0}^{[(x-y) / T]} \int_{y_{0}}^{y-y_{0}} F(y+n T-u+\Delta) F((u, y+n T]) \mathrm{d} u \\
& \leq \frac{\varepsilon}{4} \sum_{n=0}^{[(x-y) / T]} F(y+n T+\Delta) \\
& \leq \frac{\varepsilon}{4} F((y, x+T]) .
\end{aligned}
$$

By now applying Lemma 4 , we obtain $I_{1} \leq(\varepsilon / 2) F((y, x])$ for sufficiently large $y_{0}$. Second, since $\int_{0}^{\infty} \bar{F}(u) \mathrm{d} u<\infty$, for sufficiently large $y_{0}$ we have

$$
I_{2} \leq F((y, x]) \int_{y_{0}}^{y-y_{0}} \bar{F}(y-u) \mathrm{d} u \leq F((y, x]) \int_{y_{0}}^{\infty} \bar{F}(u) \mathrm{d} u \leq \frac{\varepsilon}{2} F((y, x]) .
$$


This proves (9). To prove (10), assume that (9) holds with $y_{0}$ replaced by $y_{1}$ and $\varepsilon$ replaced by $\varepsilon / 2$. Then

$$
\begin{aligned}
\left(\int_{0}^{y_{1}}+\int_{y-y_{1}}^{y}\right) F((y-u, x-u]) F((u, x]) \mathrm{d} u & \leq 2 F\left(\left(y-y_{1}, x\right]\right) \int_{0}^{y_{1}} \bar{F}(u) \mathrm{d} u \\
& \leq 2 m_{+} F\left(\left(y-y_{1}, x\right]\right) .
\end{aligned}
$$

By Lemma 4, for sufficiently large $y_{0}, 2 m_{+} F\left(\left(y-y_{1}, x\right]\right) \leq\left(2 m_{+}+\varepsilon / 2\right) F((y, x])$. This gives the upper bound in (10). Similarly, for the lower bound,

$$
\begin{aligned}
& \left(\int_{0}^{y_{1}}+\int_{y-y_{1}}^{y}\right) F((y-u, x-u]) F((u, x]) \mathrm{d} u \\
& \quad \geq 2 F\left(\left(y, x-y_{1}\right]\right)\left(\int_{0}^{y_{1}} \bar{F}(u) \mathrm{d} u-y_{1} \bar{F}\left(y_{1}\right)\right) .
\end{aligned}
$$

First we increase $y_{1}$ to ensure that

$$
\int_{0}^{y_{1}} \bar{F}(u) \mathrm{d} u-y_{1} \bar{F}\left(y_{1}\right) \geq m_{+}(1-\varepsilon) .
$$

Then we choose $y_{0}$ according to Lemma 4 to obtain

$$
F\left(\left(y-y_{1}, x\right]\right) \geq(1-\varepsilon) F((y, x])
$$

Proof of Lemma 6. By Lemma 1,

$$
\begin{aligned}
\int_{h(x)}^{x / 2} F((y, x]) F(x-y+(0, n T]) \mathrm{d} y & =\sum_{k=0}^{n-1} \int_{h(x)}^{x / 2} F((y, x]) F(x-y+k T+\Delta) \mathrm{d} y \\
& =o(1) \sum_{k=0}^{n-1} F(x+k T+\Delta) \\
& =o(1) F(x+(0, n T]) .
\end{aligned}
$$

Applying Lemma 1 again, we obtain $F \in \delta \tau_{\Delta}^{*}$ with $\Delta=(0, n T]$.

Proof of Lemma 7. By taking $x=\infty$ in Lemma 5, we immediately obtain our assertion.

Proof of Lemma 8. Let $\xi_{1}$ and $\xi_{2}$ be independent random variables with common distribution $F$. Then

$$
\begin{aligned}
(F * F)(x+\Delta)= & \mathrm{P}\left\{\xi_{1}+\xi_{2} \in x+\Delta\right\} \\
= & 2 \mathrm{P}\left\{\xi_{1} \leq h(x), \xi_{1}+\xi_{2} \in x+\Delta\right\} \\
& +\mathrm{P}\left\{\xi_{1}>h(x), \xi_{2}>h(x), \xi_{1}+\xi_{2} \in x+\Delta\right\} \\
= & : 2 I_{1}(x)+I_{2}(x) .
\end{aligned}
$$


From the definition of $h(x)$, it follows that $I_{1}(x) \sim 2 F(x+\Delta)$. Since $F \in \mathcal{L}_{\Delta}$,

$$
\begin{aligned}
I_{2}(x) & =\int_{h(x)}^{x-h(x)+T} F(\mathrm{~d} y) F(x-y+\Delta) \\
& \sim \frac{1}{T} \int_{h(x)}^{x-h(x)+T} \mathrm{~d} y F(y+\Delta) F(x-y+\Delta) \\
& \leq \frac{1}{T} \int_{h(x)}^{x-h(x)+T} \mathrm{~d} y F((y, x]) F(x-y+\Delta) \\
& =o(F(x+\Delta)),
\end{aligned}
$$

by Lemma 1 . Therefore, $F \in s_{\Delta}$.

Proof of Lemma 9. From the assumptions of Lemma 9, we conclude that $0.5 M_{1} \leq$ $G((y, x]) / F((y, x]) \leq 2 M_{2}$ for all sufficiently large $y \leq x / 2$. Suppose that $F \in \delta \tau_{\Delta}^{*}$. Then $G$ has finite expectation. Since $F, G \in \mathscr{L}_{\Delta}$, there exists a function $h(x) \rightarrow \infty$ such that (2) and the equivalent relation for $G$ hold uniformly in $|t| \leq h(x)$. Thus,

$$
\int_{h(x)}^{x / 2} \frac{G(x-y+\Delta)}{G(x+\Delta)} G((y, x]) \mathrm{d} y \leq 4 \frac{M_{2}^{2}}{M_{1}} \int_{h(x)}^{x / 2} \frac{F(x-y+\Delta)}{F(x+\Delta)} F((y, x]) \mathrm{d} y \rightarrow 0
$$

as $x \rightarrow \infty$.

Therefore, by Lemma $1, G \in \delta \tau_{\Delta}^{*}$.

\section{Acknowledgements}

The authors would like to thank Serguei Foss for drawing attention to this problem and inspiring discussions. We are grateful to Onno Boxma and Bert Zwart for many useful comments and suggestions. The authors are also grateful to the anonymous referee for a careful reading of the paper which resulted in many valuable remarks.

The research of Denis Denisov was partially supported by the Dutch BSIK/BRICKS project and the EURO-NGI project. The research of Vsevolod Shneer was supported by a James Watt Scholarship from the Heriot-Watt University, a United Kingdom Overseas Research Scholarship, and the EURO-NGI project.

\section{References}

[1] Asmussen, S. (1998). Subexponential asymptotics for stochastic processes: extremal behaviour, stationary distributions and first passage probabilities. Ann. Appl. Prob. 8, 354-374.

[2] Asmussen, S. (2003). Applied Probability and Queues, 2nd edn. Springer, New York.

[3] Asmussen, S., Foss, S. And Korshunov, D. (2003). Asymptotics for sums of random variables with local subexponential behaviour. J. Theoret. Prob. 16, 489-518.

[4] Asmussen, S. et al. (2002). A local limit theorem for random walk maxima with heavy tails. Statist. Prob. Lett. 56, 399-404.

[5] Bingham, N. H., Goldie, C. M. and Teugels, J. L. (1989). Regular Variation. Cambridge University Press.

[6] Borovkov, A. A. (2004). On the asymptotics of distributions of first-passage times. Math. Notes 75, $23-37$.

[7] Chistyakov, V. P. (1964). A theorem on sums of independent random positive variables and its applications to branching processes. Theory Prob. Appl. 9, 710-718.

[8] Denisov, D. (2005). A note on the asymptotics for the maximum on a random time interval of a random walk. Markov Process. Relat. Fields 11, 165-169.

[9] Denisov, D., Foss, S. And Korshunov, D. (2004). Tail asymptotics for the supremum of a random walk when the mean is not finite. Queueing Systems Theory Appl. 46, 15-33. 
[10] Feller, W. (1971). An Introduction to Probability Theory and Its Applications, Vol. 2, 2nd edn. John Wiley, New York.

[11] Foss, S. AND ZACHARY, S. (2003). The maximum on a random time interval of a random walk with long-tailed increments and negative drift. Ann. Appl. Prob. 13, 37-53.

[12] Foss, S., Palmowski, Z. and Zachary, S. (2005). The probability of exceeding a high boundary on a random time interval for a heavy-tailed random walk. Ann. Appl. Prob. 15, 1936-1957.

[13] Heath, D., Resnick, S. and Samorodnitsky, G. (1997). Patterns of buffer overflow in a class of queues with long memory in the input stream. Ann. Appl. Prob. 7, 1021-1057.

[14] Klüppelberg, C. (1988). Subexponential distributions and integrated tails. J. Appl. Prob. 25, 132-141.

[15] KlüPPElBerg, C. (2004). Subexponential distributions. In Encyclopedia of Actuarial Science, eds B. Sundt and J. Teugels, John Wiley, Chichester.

[16] Pitman, E. J. G. (1980). Subexponential distribution functions. J. Austral. Math. Soc. A 29, 337-347.

[17] Zwart, A. P. (2001). Tail asymptotics for the busy period in the GI/G/1 queue. Math. Operat. Res. 26, 485-493. 